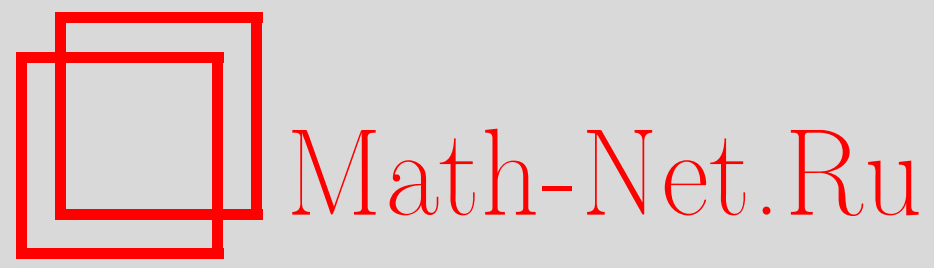

Д. А. Молодцов, Экстремальные множества семейства интервалов, Нечеткие системы и мягкие вычисления, 2018, том 13, выпуск 1, 5-15

DOI: https://doi.org/10.26456/fssc38

Использование Общероссийского математического портала Math-Net.Ru подразумевает, что вы прочитали и согласны с пользовательским соглашением

http://www.mathnet.ru/rus/agreement

Параметры загрузки:

IP: 54.197 .130 .99

26 апреля 2023 г., 15:22:29 
УДК 519.7

\section{ЭКСТРЕМАЛЬНЫЕ МНОЖЕСТВА СЕМЕЙСТВА ИНТЕРВАЛОВ}

Молодцов Д.А.

Вычислительный центр им. А.А. Дородницына Российской академии наук Федерального исследовательского центра «Информатика и управление»

Российской академии наук, г. Москва

Поступила в редакцию 08.02.2018, после переработки 01.03.2018.

Для произвольного семейства интервалов введены четыре экстремальных множества интервалов, которые представляют собой множества недоминируемых интервалов для четырех основных отношений. Изучаются свойства этих экстремальных множеств. Для каждого из этих множеств найдены еще четыре экстремальных множества и установлены связи между этими вторыми экстремальными множествами.

Ключевые слова: экстремальные множества, семейства интервалов, вторые экстремальные множества.

Нечеткие системы и мягкие вычисления. 2018. Т. 13, № 1. С. 5-15. https://doi.org/10.26456/fssc38

\section{1. Введение}

Во многих областях науки и техники используются разнообразные скалярные величины. Для приложений часть из таких величин получает свои численные значения в результате измерений. Поскольку все измерения имеют некоторую точность, то, вообще говоря, результатом измерения является интервал вещественной оси. Различные измерения одного и того же объекта дают разные интервалы. Таким образом получается, что процесс измерения некоторой величины дает семейство интервалов.

Для обработки этого семейства интервалов с целью получения числовой оценки значения (и получения единой оценки погрешности) обычно применяются различные статистические методы [1-3]. Естественно, что такого типа подходы основаны на гипотезе о случайной природе наблюдаемого разброса значений интервалов.

Если не делать предположения о случайной природе измерений, то возникает необходимость работать с семейством интервалов, как с единой совокупностью, описывающей данный объект. Естественно возникают вопросы о том, как понимать близость, эквивалентность, аппроксимацию, экстремальные значения и другие аналогичные понятия для семейств интервалов.

Еще одним источником возникновения семейства интервалов, как объекта изучения, является интервальный анализ [4,5]. Если рассмотреть функцию, принимающую интервальные значения, то множество ее значений дает семейство интервалов. Для задач оптимизации здесь естественно возникает вопрос об изучении экстремальных значений полученного семейства интервалов. 
Семейства интервалов возникают и в теории мягких множеств [6].

Приведенные задачи и являются теми мотивирующими факторами к изучению свойств семейств интервалов.

\section{2. Интервалы}

Приведем список основных обозначений, операций и отношений, которые будут использованы далее.

$\mathbb{R}$ - множество действительных чисел. Под замкнутым интервалом $u$ множества действительных чисел понимается множество вида $u=\{x \in R \mid a \leqslant x \leqslant b\}$ для некоторых действительных чисел $a, b \in R$ таких, что $a \leqslant b$. Обозначим через $\mathbb{I} \mathbb{R}$ - множество замкнутых интервалов множества $R$. Если $u \in \mathbb{I}$, то через $\underline{u}$ будем обозначать наименьшее число интервала $u$, а через $\bar{u}$ - наибольшее число интервала $u$. Таким образом $u=[\underline{u}, \bar{u}]$.

Под суммой двух интервалов $u, v \in \mathbb{I} \mathbb{R}$ понимается интервал

$$
u+v=[\underline{u}+\underline{v}, \bar{u}+\bar{v}] .
$$

Обозначения для основных отношений между двумя интервалами $u, v \in \mathbb{R}$

$$
\begin{gathered}
u \geqslant v \Longleftrightarrow \underline{u} \geqslant \underline{v}, \bar{u} \geqslant \bar{v}, \quad u \leqslant v \Longleftrightarrow v \geqslant u, \\
u>v \Longleftrightarrow(u \geqslant v) \bigwedge(u \neq v), \quad u<v \Longleftrightarrow v>u, \\
u \supseteq v \Longleftrightarrow \underline{u} \leqslant \underline{v}, \bar{u} \geqslant \bar{v}, \quad u \subseteq v \Longleftrightarrow v \supseteq u, \\
u \supset v \Longleftrightarrow(u \supseteq v) \bigwedge(u \neq v), \quad u \subset v \Longleftrightarrow v \supset u .
\end{gathered}
$$

Для любых двух интервалов $u, v \in \mathbb{R} \mathbb{R}$ справедливо, по крайней мере, одно из отношений

$$
u \geqslant v, u \leqslant v, u \supseteq v, u \subseteq v .
$$

Обозначим множество нестрогих отношений для интервалов $\operatorname{Lax} R=\{\subseteq, \supseteq$, $\leqslant, \geqslant\}$. Для отношений из этого множества введем операцию сопряжения Pair : Lax $R \rightarrow \operatorname{Lax} R$, заданную равенствами

$$
\text { Pair }(\subseteq)=\supseteq, \quad \text { Pair }(\supseteq)=\subseteq, \quad \text { Pair }(\leqslant)=\geqslant, \quad \text { Pair }(\geqslant)=\leqslant .
$$

Пары отношений $\{\subseteq, \supseteq\}$ и $\{\leqslant, \geqslant\}$ будем называть оппозиционными друг к другу, а отношения в парах будем называть родственными.

\section{3. Отношения и операции для семейств интервалов}

Множество всех семейств интервалов обозначим $2^{\mathbb{R}}$. Отметим, что под семейством понимается совокупность различных интервалов.

Семейство интервалов $A \in 2^{\mathbb{R}}$ называется ограниченным, если существует интервал, которому принадлежат все интервалы из семейства $A$.

Семейство интервалов $A \in 2^{\mathbb{R}}$ называется конечным, если его мощность конечна.

Введем отображение $\pi: \mathbb{R} \rightarrow \mathbb{R}^{2}$, где $\pi(u)=(\underline{u}, \bar{u}) \in \mathbb{R}^{2}$. Тогда $\pi A \subseteq \mathbb{R}^{2}$.

Отношения для интервалов при отображении $\pi$ индуцируют отношения для точек двумерной плоскости. Будем использовать для них те же обозначения, что и для интервалов. 
Семейство интервалов $A \in 2^{\mathbb{R R}}$ называется замкнутым, если подмножество $\pi A$ является замкнутым множеством в $R^{2}$.

Семейство интервалов назовем компактным, если оно ограничено и замкнуто.

Если задано семейство $A \in 2^{\mathbb{I R}}$, то будем использовать следующие обозначения

$$
\underline{A}=\{\underline{u} \in R \mid u \in A\}, \quad \bar{A}=\{\bar{u} \in R \mid u \in A\} .
$$

Введем несколько отношений для семейств интервалов, используя основные отношения для интервалов.

Определение 3.1. Пусть $A, B \in 2^{\mathbb{I R}}$ два непустых семейства интервалов и $\theta \in \mathrm{LaxR}$. A $\theta$-аппроксимирует $B$, если для любого интервала $b \in B$ существует интервал $а \in A$ такой, что а $\theta$ b. Обозначение $A \theta B$.

Все эти четыре отношения являются рефлексивными и транзитивными, но не антисимметричными.

Отметим, что если $A \subseteq B$, то это не означает, что каждый интервал из семейства $A$ уже некоторого интервала из семейства $B$. Поэтому введем еще четыре отношения, усиливающие введенные в определении 3.1 .

Определение 3.2. Пусть $A, B \in 2^{\mathbb{R} R}$ два непустых семейства интервалов и $\theta \in \mathrm{LaxR}$. A строго $\theta$-аппроксимирует $B$, если $A \theta B$ и $B \operatorname{Pair}(\theta) A$. Обозначение $A \stackrel{!}{!} B$.

Все эти четыре строгих отношения также являются рефлексивными и транзитивными, но не антисимметричными.

На базе введенных отношений построим серию отношений эквивалентности.

Определение 3.3. Пусть $A, B \in 2^{\mathbb{R} R}$ два непустых семейства интервалов и $\theta \in \mathrm{LaxR}$.

1. $A \theta$-эквивалентно $B$, если $A \theta B$ и $B \theta A$. Обозначение $A\langle\theta\rangle B$.

2. $A$ строго $\theta$-эквивалентно $B$, если $A ! \theta B$ и $B ! \theta A$. Обозначение $A\langle! \theta\rangle B$.

3. $A$ эквивалентно $B$, если $A\langle! \leq\rangle B$ и $A\langle! \subseteq\rangle B$. Обозначение $A \approx B$.

Отметим, что различных здесь только семь отношений. Первый пункт дает четыре отношения, а второй пункт только два, так как отношения в парах $\{\langle! \subseteq\rangle,\langle! \supseteq\rangle\}$ и $\{\langle! \leq\rangle,\langle! \geq\rangle\}$ совпадают.

Определение 3.4. Семейство интервалов $A \in 2^{\mathbb{I R}}$ называется продолжением семейства интервалов $B \in 2^{\mathbb{R}}$, если семейство $A$ иире семейства $B$, то есть $A \supseteq B$.

Непосредственно из определения продолжения следует утверждение.

Утверждение 3.1. Пусть $A, B \in 2^{\mathbb{R}}$ два непустых семейства интервалов и $A \supseteq B$ тогда

$$
A \supseteq B, \quad A \subseteq B, \quad A \geqslant B, \quad A \leqslant B .
$$

Суммой двух непустых семейств интервалов $A, B \in 2^{\mathbb{R}}$ назовем следующее семейство интервалов

$$
A+B=\{u \in \mathbb{I} \mid \exists a \in A, \quad \exists b \in B: u=a+b\} .
$$


Если хотя бы одно из семейств пусто, то сумма не определена.

Сумма конечного числа непустых семейств определяется по индукции.

Объединением двух семейств интервалов $A, B \in 2^{\mathbb{R}}$ назовем семейство $A \cup B$ интервалов, состоящих из интервалов, входящих по крайней мере в одно из семейств $A, B \in 2^{\mathbb{I R}}$. Аналогично определяется объединение любого множества семейств интервалов.

\section{4. Экстремальные множества семейства интервалов}

Для множества вещественных чисел экстремальными множествами, а точнее числами, являются верхняя и нижняя грани. Введем четыре экстремальных множества для семейства интервалов $A \in 2^{\mathbb{I R}}$. Пусть $\theta \in \mathrm{LaxR}$.

$$
\operatorname{Extr}(A, \theta)=\{a \in A \mid \nexists b \in A:(b \theta a) \bigwedge(a \neq b)\} .
$$

По сути, экстремальные множества - это множества Парето для соответствующих отношений, и поэтому для их нахождения можно применять весь арсенал методов, разработанных в теории многокритериальных задач [7].

Следующее простое утверждение, использующее один из методов многокритериальной оптимизации, является основным техническим инструментом для дальнейших построений.

Утверждение 4.1. Пусть семейство интервалов $A \in 2^{\mathbb{R}}$ компактно u $\theta \in \operatorname{LaxR}$. Тогда для любого интервала $u \in A$ существует интервал $v \in \operatorname{Extr}(A, \theta)$ maкой, что $v \theta u$.

Замечание 1. Для множества $\operatorname{Extr}(A, \subseteq)$ для справедливости утверждения достаточно только замкнутости семейства $A \in 2^{\mathbb{R} R}$.

Доказательство. Рассмотрим подмножество евклидовой плоскости

$$
D(A, u, \theta)=\{v \in \pi A \mid v \theta u\} .
$$

Это множество не пусто, замкнуто и ограничено. На множестве $D(A, u, \subseteq)$ находим наименьшее значение функции $\bar{v}-\underline{v}$, на множестве $D(A, u, \supseteq)$ находим наибольшее значение функции $\bar{v}-\underline{v}$, на множестве $D(A, u, \geqslant)$ находим наибольшее значение функции $\bar{v}+\underline{v}$, на множестве $D(A, u, \leqslant)$ находим наименьшее значение функции $\bar{v}+\underline{v}$. Полученные реализации дают точки плоскости для формирования требуемых интервалов. Утверждение доказано.

Приведем ряд элементарных свойств введенных экстремальных семейств интервалов. Сначала опишем результат применения экстремальных множеств к сумме и объединению семейств интервалов.

Утверждение 4.2. Пусть непустые семейства интервалов $A, B \in 2^{\mathbb{R}}$ компактны и пусть $\theta \in \mathrm{LaxR}$. Тогда

1. $\operatorname{Extr}(A+B, \theta) \subseteq \operatorname{Extr}(A, \theta)+\operatorname{Extr}(B, \theta)$,

2. $\operatorname{Extr}(A+B, \theta)=\operatorname{Extr}(\operatorname{Extr}(A, \theta)+\operatorname{Extr}(B, \theta), \theta)$,

3. $\operatorname{Extr}(A \bigcup B, \theta)=\operatorname{Extr}(\operatorname{Extr}(A, \theta) \bigcup \operatorname{Extr}(B, \theta), \theta)$. 
Доказательство элементарно.

Теперь установим связь между отношениями доминирования двух семейств интервалов и отношениями доминирования их экстремальных множеств.

Утверждение 4.3. Пусть непустые семейства интервалов $A, B \in 2^{\mathbb{I R}}$ компактны и пусть $\theta \in \mathrm{LaxR}$. Тогда

1. $A \theta B$ тогда и только тогда, когда $\operatorname{Extr}(A, \theta) \theta \operatorname{Extr}(B, \theta)$,

2. $A[! B$ тогда и толъко тогда, когда $\operatorname{Extr}(A, \theta) \theta \operatorname{Extr}(B, \theta)$ и $\operatorname{Extr}(B, \operatorname{Pair}(\theta)) \quad \operatorname{Pair}(\theta) \operatorname{Extr}(A, \operatorname{Pair}(\theta))$.

Доказательство очевидно.

Следующее утверждение устанавливает аналогичную связь для различных отношений эквивалентности.

Утверждение 4.4. Пусть непустые семейства интервалов $A, B \in 2^{\mathbb{R}}$ компактны и пусть $\theta \in \mathrm{LaxR}$. Тогда

1. $A\langle\theta\rangle B$ тогда и только тогда, когда $\operatorname{Extr}(A, \theta)=\operatorname{Extr}(B, \theta)$,

2. $A\langle! \theta\rangle B$ тогда и только тогда, когда

$$
\operatorname{Extr}(A, \theta)=\operatorname{Extr}(B, \theta), \quad \operatorname{Extr}(A, \operatorname{Pair}(\theta))=\operatorname{Extr}(B, \operatorname{Pair}(\theta)),
$$

3. $A \approx B$ тогда и толъко тогда, когда

$$
\begin{aligned}
& \operatorname{Extr}(A, \subseteq)=\operatorname{Extr}(B, \subseteq), \quad \operatorname{Extr}(A, \supseteq)=\operatorname{Extr}(B, \supseteq), \\
& \operatorname{Extr}(A, \leqslant)=\operatorname{Extr}(B, \leqslant), \quad \operatorname{Extr}(A, \geqslant)=\operatorname{Extr}(B, \geqslant) .
\end{aligned}
$$

Доказательство элементарно.

Следующее утверждение устанавливает эквивалентность различного типа между семейством интервалов и его экстремальными множествами.

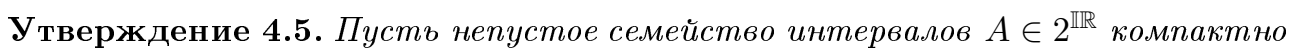
$u \theta \in \operatorname{LaxR}$. Тогда

1. $\operatorname{Extr}(A, \theta)\langle\theta\rangle A$,

2. $(\operatorname{Extr}(A, \theta) \cup \operatorname{Extr}(A, \operatorname{Pair}(\theta)))\langle! \theta\rangle A$,

3. $(\operatorname{Extr}(A, \subseteq) \cup \operatorname{Extr}(A, \supseteq) \cup \operatorname{Extr}(A, \leqslant) \cup \operatorname{Extr}(A, \geqslant)) \approx A$.

Доказательство очевидно.

Из этого утверждения следует, что с точностью до эквивалентности компактное семейство интервалов определяется своими четырьмя экстремальными множествами.

Следующее утверждение находит некоторые экстремальные множества от экстремальных множеств, то есть вторые экстремальные множества.

Утверждение 4.6. Если непустое семейство интервалов $A \in 2^{\mathbb{I R}}$ компактно $u \theta \in \mathrm{LaxR}$, тогда

$$
\operatorname{Extr}(\operatorname{Extr}(A, \theta), \theta)=\operatorname{Extr}(\operatorname{Extr}(A, \theta), \operatorname{Pair}(\theta))=\operatorname{Extr}(A, \theta) .
$$

Доказательство очевидно.

Утверждение 4.7. Если непустое семейство интервалов $A \in 2^{\mathbb{R}}$ компактно, тогда 
1. семейство $\operatorname{Extr}(A, \subseteq)$ вполне упорлдочено по отношению $\geqslant$,

2. семейство $\operatorname{Extr}(A, \supseteq)$ вполне упорядочено по отношению $\geqslant$,

3. семейство $\operatorname{Extr}(A, \leqslant)$ вполне упорядочено по отношению $\supseteq$,

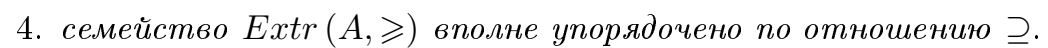

Доказательство очевидно.

Отметим известный факт из теории многокритериальных задач о том, что даже если семейство интервалов компактно, то экстремальные множества могут быть не замкнуты. Рассмотрим пример семейства интервалов. Пусть $A \in 2^{\mathbb{I R}}$ имеет следующий вид $A=\left\{[0,1],\left[\frac{1}{2}, 1\right],\left[-\frac{1}{n}, 1-\frac{1}{n}\right], n=1,2, \ldots\right\}$.

Заметим, что $A$ замкнуто и $\operatorname{Extr}(A, \subseteq)=\left\{\left[\frac{1}{2}, 1\right],\left[-\frac{1}{n}, 1-\frac{1}{n}\right], n=1,2, \ldots\right\}$, но множество $\operatorname{Extr}(A, \subseteq)$ не замкнуто. Тем не менее, наибольшее и наименьшее значения концов интервалов в экстремальных множествах достигаются. Более того, существует интервал, который своими концами реализует решение сразу двух экстремальных задач такого типа. Следующее утверждение дает оставшиеся вторые экстремальные множества.

Утверждение 4.8. Пусть непустое семейство интервалов $A \in 2^{\mathbb{I R}}$ компактно. Тогда

1. $\operatorname{Extr}(\operatorname{Extr}(A, \subseteq), \leqslant)=\left[\inf _{u \in \operatorname{Extr}(A, \subseteq)} \underline{u}, \inf _{u \in \operatorname{Extr}(A, \subseteq)} \bar{u}\right] \in \operatorname{Extr}(A, \subseteq)$,

2. $\operatorname{Extr}(\operatorname{Extr}(A, \subseteq), \geqslant)=\left[\sup _{u \in \operatorname{Extr}(A, \subseteq)} \underline{u}, \sup _{u \in \operatorname{Extr}(A, \subseteq)} \bar{u}\right] \in \operatorname{Extr}(A, \subseteq)$,

3. $\operatorname{Extr}(\operatorname{Extr}(A, \supseteq), \leqslant)=\left[\inf _{u \in \operatorname{Extr}(A, \supseteq)} \underline{u}, \inf _{u \in \operatorname{Extr}(A, \supseteq)} \bar{u}\right] \in \operatorname{Extr}(A, \supseteq)$,

4. $\operatorname{Extr}(\operatorname{Extr}(A, \supseteq), \geqslant)=\left[\sup _{u \in \operatorname{Extr}(A, \supseteq)} \underline{u}, \sup _{u \in \operatorname{Extr}(A, \supseteq)} \bar{u}\right] \in \operatorname{Extr}(A, \supseteq)$,

5. $\operatorname{Extr}(\operatorname{Extr}(A, \geqslant), \subseteq)=\left[\sup _{u \in \operatorname{Extr}(A, \geq)} \underline{u}, \inf _{u \in \operatorname{Extr}(A, \geq)} \bar{u}\right] \in \operatorname{Extr}(A, \geqslant)$,

6. $\operatorname{Extr}(\operatorname{Extr}(A, \geqslant), \supseteq)=\left[\inf _{u \in \operatorname{Extr}(A, \geq)} \underline{u}, \sup _{u \in \operatorname{Extr}(A, \geq)} \bar{u}\right] \in \operatorname{Extr}(A, \geqslant)$,

7. $\operatorname{Extr}(\operatorname{Extr}(A, \leqslant), \subseteq)=\left[\sup _{u \in \operatorname{Extr}(A, \leq)} \underline{u}, \inf _{u \in \operatorname{Extr}(A, \leq)} \bar{u}\right] \in \operatorname{Extr}(A, \leqslant)$,

8. $\operatorname{Extr}(\operatorname{Extr}(A, \leqslant), \supseteq)=\left[\inf _{u \in \operatorname{Extr}(A, \leq)} \underline{u}, \sup _{u \in \operatorname{Extr}(A, \leq)} \bar{u}\right] \in \operatorname{Extr}(A, \leqslant)$. 
Доказательство. Проведем доказательство для пункта 2. Величины $\sup _{u x \operatorname{tr}(A, \subseteq)} \underline{u}, \sup _{u \in \operatorname{Extr}(A, \subseteq)} \bar{u} \quad$ конечны в силу ограниченности семейства

$\operatorname{Extr}(A, \subseteq)$. Возьмем произвольное натуральное $k$ и тогда существует интервал $u_{k} \in \operatorname{Extr}(A, \subseteq)$ такой, что $\bar{u}_{k} \geqslant \sup _{u \in \operatorname{Extr}(A, \subseteq)} \bar{u}-\frac{1}{k}$. Если $\underline{u}_{k} \geqslant \sup _{u \in \operatorname{Extr}(A, \subseteq)} \underline{u}-\frac{1}{k}$, то положим $v_{k}=u_{k} \in \operatorname{Extr}(A, \subseteq)$, в противном случае существует интервал $v_{k} \in \operatorname{Extr}(A, \subseteq)$ такой, что $\underline{v}_{k} \geqslant \sup _{u \in \operatorname{Extr}(A, \subseteq)} \underline{u}-\frac{1}{k} \cdot$ Поскольку множество $\operatorname{Extr}(A, \subseteq)$ вполне упорядочено по отношению $\geqslant$ и выполнены неравенства $\underline{u}_{k}<\sup _{u \in \operatorname{Extr}(A, \subseteq)} \underline{u}-\frac{1}{k} \leqslant \underline{v}_{k}$, то должны также выполняться неравенства $\sup _{u \in \operatorname{Extr}(A, \subseteq)} \bar{u}-\frac{1}{k} \leqslant \bar{u}_{k} \leqslant \bar{v}_{k}$

Таким образом построена последовательность $v_{k} \in \operatorname{Extr}(A, \subseteq)$, для которой выполнены неравенства

$$
\sup _{u \in \operatorname{Extr}(A, \subseteq)} \underline{u}-\frac{1}{k} \leqslant \underline{v}_{k} \leqslant \sup _{u \in \operatorname{Extr}(A, \subseteq)} \underline{u}, \quad \sup _{u \in \operatorname{Extr}(A, \subseteq)} \bar{u}-\frac{1}{k} \leqslant \bar{v}_{k} \leqslant \sup _{u \in \operatorname{Extr}(A, \subseteq)} \bar{u} .
$$

В силу замкнутости семейства интервалов $A \in 2^{\mathbb{R}}$, эта последовательность сходится к интервалу $q=\left[\sup _{u \in \operatorname{Extr}(A, \subseteq)} \underline{u}, \sup _{u \in \operatorname{Extr}(A, \subseteq)} \bar{u}\right] \in A$. Предположим, что $q \notin \operatorname{Extr}(A, \subseteq)$. Тогда в силу утверждения 3.2 . существует интервал $w \in \operatorname{Extr}(A, \subseteq)$ такой, что $w \subset q$. Поскольку неравенство $\underline{w}>\sup _{u \in \operatorname{Extr}(A, \subseteq)} \underline{u}$ невозможно, то должны быть выполнены соотношения $\underline{w}=\underline{q}=\sup _{u \in \operatorname{Extr}(A, \subseteq)} \underline{u}$, $\bar{w}+\varepsilon=\bar{q}=\sup _{u \in \operatorname{Extr}(A, \subseteq)} \bar{u}, \varepsilon>0$.

Пусть для натурального $\mathrm{k}$ выполнено неравенство $k>\frac{1}{\varepsilon}$, тогда получаем

$$
\underline{w} \geqslant \underline{v}_{k}, \quad \bar{v}_{k} \geqslant \bar{w}+\varepsilon-\frac{1}{k}>\bar{w},
$$

то есть выполнено строгое отношение $w \subset v_{k}$. Поскольку $w, v_{k} \in \operatorname{Extr}(A, \subseteq)$, получили противоречие. Следовательно, $q \in \operatorname{Extr}(A, \subseteq)$. Поскольку для любых интервалов $u \in \operatorname{Extr}(A, \subseteq)$ выполнено неравенство $q \geqslant u$, получаем $\operatorname{Extr}(\operatorname{Extr}(A, \subseteq), \geqslant)=\{q\}$.

Проведем теперь доказательство для пункта 5. Величины $\underline{q}=\sup _{u \in \operatorname{Extr}(A, \geq)} \underline{u}$, $\bar{q}=\inf _{u \in \operatorname{Extr}(A, \geq)} \bar{u}$ конечны. Для любого натурального $\mathrm{k}$ существует интервал $u_{k} \in \operatorname{Extr}(A, \geqslant)$, для которого выполнено неравенство $\underline{u}_{k} \geqslant \underline{q}-\frac{1}{k}$. Если $\bar{u}_{k} \leqslant \bar{q}+\frac{1}{k}$, то положим $v_{k}=u_{k}$.

В противном случае, то есть, когда $\bar{u}_{k}>\bar{q}+\frac{1}{k}$, существует интервал $v_{k} \in \operatorname{Extr}(A, \geqslant)$ такой, что $\bar{v}_{k} \leqslant \bar{q}+\frac{1}{k}<\bar{u}_{k}$. Поскольку множество $\operatorname{Extr}(A, \geqslant)$ вполне упорядочено по отношению $\supseteq$ и выполнено неравенство $\bar{v}_{k}<\bar{u}_{k}$, то должны выполняться также и неравенства $\underline{v}_{k} \geqslant \underline{u}_{k} \geqslant \underline{q}-\frac{1}{k}$. Итак, существует последовательность интервалов $v_{k} \in \operatorname{Extr}(A, \geqslant)$, для которой выполнены неравенства

$$
\bar{q} \leqslant \bar{v}_{k} \leqslant \bar{q}+\frac{1}{k}, \quad \underline{q} \geqslant \underline{v}_{k} \geqslant \underline{q}-\frac{1}{k}, \quad \bar{q}+\frac{1}{k} \geqslant \bar{v}_{k} \geqslant \underline{v}_{k} \geqslant \underline{q}-\frac{1}{k} .
$$

Переходя к пределу при $k \rightarrow \infty$, получаем $\bar{q} \geqslant \underline{q}$ и последовательность интервалов $v_{k} \in \operatorname{Extr}(A, \geqslant)$ сходится к интервалу $q \underline{q}=[\underline{q}, \bar{q}]$. Предположим, что 
$q \notin \operatorname{Extr}(A, \geqslant)$, тогда из утверждения 3.2. следует, что существует интервал $w \in \operatorname{Extr}(A, \geqslant)$ такой, что $w>q$. Поскольку неравенство $\underline{w}>\underline{q}$ невозможно выполнены равенства $\underline{w}=\underline{q}$ и $\bar{w}=\bar{q}+\varepsilon$, для некоторого $\varepsilon>0$. Пусть для натурального $k$ выполнено неравенство $k>\frac{1}{\varepsilon}$, тогда получаем

$$
\underline{w} \geqslant \underline{v}_{k}, \quad \bar{w}=\bar{q}+\varepsilon \geqslant \bar{v}_{k}+\varepsilon-\frac{1}{k}>\bar{v}_{k},
$$

то есть выполнено строгое отношение $w>v_{k}$ для двух интервалов из множества $\operatorname{Extr}(A, \geqslant)$, что является противоречием. Итак, получаем $\operatorname{Extr}(\operatorname{Extr}(A, \geqslant), \subseteq)=\{q\}$. Доказательство остальных равенств аналогично. Утверждение доказано.

Оказывается, что между интервалами из предыдущего утверждения, то есть между вторыми экстремальными множествами, существуют некоторые интересные связи.

Утверждение 4.9. Пусть непустое семейство интервалов $A \in 2^{\mathbb{I R}}$ компактно. Тогда

$$
\begin{aligned}
& \text { 1. } \inf _{u \in \operatorname{Extr}(A, \subseteq)} \bar{u}=\overline{\operatorname{Extr}}(\operatorname{Extr}(A, \subseteq), \leqslant)=\overline{\operatorname{Extr}}(\operatorname{Extr}(A, \leqslant), \subseteq)=\inf _{u \in \operatorname{Extr}(A, \leq)} \bar{u}, \\
& \text { 2. } \sup _{u \in \operatorname{Extr}(A, \subseteq)} \underline{u}=\underline{\operatorname{Extr}}(\operatorname{Extr}(A, \subseteq), \geqslant)=\underline{\operatorname{Extr}}(\operatorname{Extr}(A, \geqslant), \subseteq)=\sup _{u \in \operatorname{Extr}(A, \geq)} \underline{u}, \\
& \text { 3. } \inf _{u \in \operatorname{Extr}(A, \supseteq)} \underline{u}=\underline{\operatorname{Extr}}(\operatorname{Extr}(A, \supseteq), \leqslant)=\underline{\operatorname{Extr}}(\operatorname{Extr}(A, \leqslant), \supseteq)=\inf _{u \in \operatorname{Extr}(A, \leq)} \underline{u}, \\
& \text { 4. } \sup _{u \in \operatorname{Extr}(A, \supseteq)} \bar{u}=\overline{\operatorname{Extr}}(\operatorname{Extr}(A, \supseteq), \geqslant)=\operatorname{Extr}(\operatorname{Extr}(A, \geqslant), \supseteq)=\sup _{u \in \operatorname{Extr}(A, \geq)} \bar{u}, \\
& \text { 5. } \inf _{u \in \operatorname{Extr}(A, \subseteq)} \underline{u}=\underline{\operatorname{Extr}}(\operatorname{Extr}(A, \subseteq), \leqslant) \geqslant \underline{\operatorname{Extr}}(\operatorname{Extr}(A, \leqslant), \subseteq)=\sup _{u \in \operatorname{Extr}(A, \leq)} \underline{u}, \\
& \text { 6. } \sup _{u \in \operatorname{Extr}(A, \subseteq)} \bar{u}=\overline{\operatorname{Extr}}(\operatorname{Extr}(A, \subseteq), \geqslant) \leqslant \overline{\operatorname{Extr}}(\operatorname{Extr}(A, \geqslant), \subseteq)=\inf _{u \in \operatorname{Extr}(A, \geq)} \bar{u}, \\
& \text { 7. } \inf _{u \in \operatorname{Extr}(A, \supseteq)} \bar{u}=\overline{\operatorname{Extr}}(\operatorname{Extr}(A, \supseteq), \leqslant) \geqslant \overline{\operatorname{Extr}}(\operatorname{Extr}(A, \leqslant), \supseteq)=\sup _{u \in \operatorname{Extr}(A, \leq)} \bar{u}, \\
& \text { 8. } \sup _{u \in \operatorname{Extr}(A, \supseteq)} \underline{u}=\underline{\operatorname{Extr}}(\operatorname{Extr}(A, \supseteq), \geqslant) \leqslant \underline{\operatorname{Extr}}(\operatorname{Extr}(A, \geqslant), \supseteq)=\inf _{u \in \operatorname{Extr}(A, \geq)} \underline{u} .
\end{aligned}
$$

Доказательство. Пункт 1. Из утверждения 4.1. следует, что для интервала

$$
\operatorname{Extr}(\operatorname{Extr}(A, \subseteq), \leqslant)=\left[\inf _{u \in \operatorname{Extr}(A, \subseteq)} \underline{u}, \inf _{u \in \operatorname{Extr}(A, \subseteq)} \bar{u}\right] \in \operatorname{Extr}(A, \subseteq)
$$

существует интервал $a \in \operatorname{Extr}(A, \leqslant)$, такой, что $a \leqslant \operatorname{Extr}(\operatorname{Extr}(A, \subseteq), \leqslant)$, то есть

$$
\underline{a} \leqslant \underline{\operatorname{Extr}}(\operatorname{Extr}(A, \subseteq), \leqslant), \quad \bar{a} \leqslant \overline{\operatorname{Extr}}(\operatorname{Extr}(A, \subseteq), \leqslant) .
$$

Отсюда получаем

$$
\overline{\operatorname{Extr}}(\operatorname{Extr}(A, \leqslant), \subseteq)=\inf _{u \in \operatorname{Extr}(A, \leq)} \bar{u} \leqslant \bar{a} \leqslant \overline{\operatorname{Extr}}(\operatorname{Extr}(A, \subseteq), \leqslant) .
$$


Предположим, что неравенство строгое, то есть выполнено

$$
\overline{\operatorname{Extr}}(\operatorname{Extr}(A, \leqslant), \subseteq)=\inf _{u \in \operatorname{Extr}(A, \leq)} \bar{u}<\overline{\operatorname{Extr}}(\operatorname{Extr}(A, \subseteq), \leqslant) .
$$

Тогда для интервала

$$
\operatorname{Extr}(\operatorname{Extr}(A, \leqslant), \subseteq)=\left[\sup _{u \in \operatorname{Extr}(A, \leq)} \underline{u}, \inf _{u \in \operatorname{Extr}(A, \leq)} \bar{u}\right] \in \operatorname{Extr}(A, \leqslant)
$$

существует интервал $b \in \operatorname{Extr}(A, \subseteq)$ такой, что $b \subseteq \operatorname{Extr}(\operatorname{Extr}(A, \leqslant), \subseteq)$, то есть

$$
\underline{b} \geqslant \underline{\operatorname{Extr}}(\operatorname{Extr}(A, \leqslant), \subseteq), \quad \bar{b} \leqslant \overline{\operatorname{Extr}}(\operatorname{Extr}(A, \leqslant), \subseteq) .
$$

Отсюда имеем

$$
\bar{b} \leqslant \overline{\operatorname{Extr}}(\operatorname{Extr}(A, \leqslant), \subseteq)<\overline{\operatorname{Extr}}(\operatorname{Extr}(A, \subseteq), \leqslant)=\inf _{u \in \operatorname{Extr}(A, \subseteq)} \bar{u} .
$$

Получили противоречие. Следовательно, пункт 1 доказан.

Возьмем два интервала

$$
\operatorname{Extr}(\operatorname{Extr}(A, \subseteq), \leqslant) \in \operatorname{Extr}(A, \subseteq), \quad \operatorname{Extr}(\operatorname{Extr}(A, \leqslant), \subseteq) \in \operatorname{Extr}(A, \leqslant) .
$$

Если бы было выполнено неравенство

$$
\underline{\operatorname{Extr}}(\operatorname{Extr}(A, \subseteq), \leqslant)<\underline{\operatorname{Extr}}(\operatorname{Extr}(A, \leqslant), \subseteq),
$$

то, учитывая доказанное равенство

$$
\overline{\operatorname{Extr}}(\operatorname{Extr}(A, \subseteq), \leqslant)=\overline{\operatorname{Extr}}(\operatorname{Extr}(A, \leqslant), \subseteq),
$$

получаем, что справедливо отношение

$$
\operatorname{Extr}(\operatorname{Extr}(A, \leqslant), \subseteq) \subset \operatorname{Extr}(\operatorname{Extr}(A, \subseteq), \leqslant)
$$

и тогда интервал $\operatorname{Extr}(\operatorname{Extr}(A, \subseteq), \leqslant)$ не мог бы принадлежать множеству $\operatorname{Extr}(A, \subseteq)$. Полученное противоречие доказывает пункт 5 . Остальные пункты доказываются аналогично.

\section{Заключение}

Для произвольного семейства интервалов были введены четыре экстремальных множества интервалов, которые представляют собой множества недоминируемых интервалов для четырех основных отношений $\{\subseteq, \supseteq, \leqslant, \geqslant\}$. Для компактных семейств интервалов все эти экстремальные множества не пусты. К полученным четырем экстремальным множествам можно применить тот же подход и найти для каждого из них еще четыре экстремальных множества. Вторые экстремальные множества по родственным отношениям совпадают с соответствующими первыми экстремальными множествами. Вторые экстремальные множества по оппозиционным отношениям состоят из одного интервала и эти интервалы обладают интересными свойствами. Во-первых, каждый из этих интервалов является реализацией сразу двух оптимизационных задач для концов интервала по первому экстремальному множеству. Во-вторых, эти интервалы тесно взаимосвязаны друг с другом, часть концов у них попарно совпадают, а для других концов выполнены определенные неравенства. 


\section{Список литературы}

[1] Бендат Дж., Пирсол А. Прикладной анализ случайных данных. М.: Мир, 1989. $540 \mathrm{c}$.

[2] Кунце Х.-И. Методы физических измерений. М.: Мир, 1989.

[3] Тейлор Дж. Введение в теорию ошибок. М.: Мир, 1985.

[4] Шарый С.П. Конечномерный интервальный анализ. Новосибирск: XYZ, 2017.

[5] Калмыков С.А., Шокин Ю.И., Юлдашев 3.Х. Методы интервального анализа. Новосибирск: Наука, 1986.

[6] Молодцов Д.А. Теория мягких множеств. М.: УРСС, 2004.

[7] Подиновский В.В., Ногин В.Д. Парето-оптимальные решения многокритериальных задач. М.: Наука, 1982.

\section{Образец цитирования}

Молодцов Д.А. Экстремальные множества семейства интервалов // Нечеткие системы и мягкие вычисления. 2018. Т. 13, № 1. С. 5-15. https://doi.org/10.26456/ fssc 38

\section{Сведения об авторах}

\section{1. Молодцов Дмитрий Анатольевич}

старший научный сотрудник Вычислительного центра им. А.А. Дородницына Российской академии наук Федерального исследовательского центра «Информатика и управление» Российской академии наук.

Россия, 119311, г. Москва, ул. Вавилова, д. 40, ВЦРАН. 


\title{
EXTREMAL SETS OF THE FAMILY OF INTERVALS
}

\author{
Molodtsov Dmitriy Anatolievich \\ Senior researcher at Institution of Russian Academy of Sciences \\ Dorodnicyn Computing Centre of RAS \\ Russia, 119333, Moscow, 40 Vavilova str., CC RAS.
}

Received 08.02.2018, revised 01.03.2018.

For an arbitrary family of intervals, four extremal sets of intervals are introduced, which are sets of non-dominated intervals for four basic relations for intervals. The properties of these extremal sets are studied. For each of these sets, four more extremal sets were found and connections between these second extremal sets were established.

Keywords: extremal sets, families of intervals, second extremal sets.

Nechetkie Sistemy i Myagkie Vychisleniya [Fuzzy Systems and Soft Computing], 2018, vol. 13, no. 1, pp. 5-15. https://doi.org/10.26456/ fssc 38

\section{References}

[1] Bendat Dzh., Pirsol A. Prikladnoj Analiz Sluchajnykh Dannykh [Applied Analysis of Random Data]. Mir Publ., Moscow, 1989. 540 p. (in Russian)

[2] Kuntse Kh.-I. Metody Fizicheskikh Izmerenij [Methods of Physical Measurements]. Mir Publ., Moscow, 1989. (in Russian)

[3] Teylor Dzh. Vvedenie v Teoriyu Oshibok [Introduction to the Theory of Errors]. Mir Publ., Moscow, 1985. (in Russian)

[4] Shariy S.P. Konechnomernyj Interval'nyj Analiz [Finite-Dimensional Interval Analysis]. XYZ Publ., Novosibirsk, 2017. (in Russian)

[5] Kalmykov S.A., Shokin Yu.I., Yuldashev Z.Kh. Metody Interval'nogo Analiza [Methods of Interval Analysis]. Nauka Publ., Novosibirsk, 1986. (in Russian)

[6] Molodtsov D.A. Teoriya Myagkikh Mnozhestv [The Theory of Soft Sets]. Editorial URSS Publ., Moscow, 2004. (in Russian)

[7] Podinovskiy V.V., Nogin V.D. Pareto-Optimal'nye Resheniya Mnogokriterial'nykh Zadach [Pareto-Optimal Solutions of Multicriteria Problems]. Nauka Publ., Moscow, 1982. (in Russian)

\section{Citation}

Molodtsov D.A. Extremal sets of the family of intervals. Nechetkie Sistemy $i$ Myagkie Vychisleniya [Fuzzy Systems and Soft Computing], 2018, vol. 13, no. 1, pp. 5-15. (in Russian) https://doi.org/10.26456/fssc38 